\title{
Article
}

\section{Effect of velocity-based loading on acceleration kinetics and kinematics during sled towing}

Bentley, Ian, Edmundson, Christopher James, Sinclair, Jonathan Kenneth, Atkins, Stephen and Metcalfe, John

Available at http://clok.uclan.ac.uk/23758/

Bentley, Ian ORCID: 0000-0002-9086-2338, Edmundson, Christopher James ORCID: 0000-0003-2275-7584, Sinclair, Jonathan Kenneth ORCID: 0000-00022231-3732, Atkins, Stephen and Metcalfe, John ORCID: 0000-0002-8414-978X (2018) Effect of velocity-based loading on acceleration kinetics and kinematics during sled towing. Journal of Strength \& Conditioning Research . ISSN 1064-8011

It is advisable to refer to the publisher's version if you intend to cite from the work. http://dx.doi.org/10.1519/JSC.0000000000002850

For more information about UCLan's research in this area go to

http://www.uclan.ac.uk/researchgroups/ and search for < name of research Group>.

For information about Research generally at UCLan please go to http://www.uclan.ac.uk/research/

All outputs in CLoK are protected by Intellectual Property Rights law, including Copyright law. Copyright, IPR and Moral Rights for the works on this site are retained by the individual authors and/or other copyright owners. Terms and conditions for use of this material are defined in the policies page.

\section{CLoK}

Central Lancashire online Knowledge www.clok.uclan.ac.uk

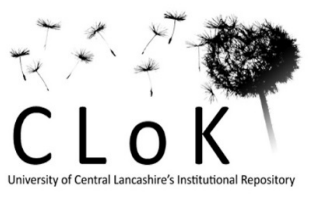




\section{ABSTRACT}

5

6 Sled towing (ST) provides an external load in the form of a sled towed via a shoulder

7 or waist harness and cord, behind the athlete. Loading strategies have varied greatly

\section{during sled towing} between studies and despite many investigations there is little agreement on the optimum sled loading to develop the acceleration phase. The aim of this study was to investigate the kinetics and kinematics of velocity-based ST during the acceleration phase of sprinting. Twelve academy rugby league players performed a series of $6 \mathrm{~m}$ sprints in different conditions; uninhibited, $10 \%, 15 \%$ and $20 \%$ velocity decrement ( $\left.V_{\text {Dec }}\right)$. Sagittal plane kinematics and kinetic measures were examined using one-way repeated measures analysis of variance. Results indicated that ST affected trunk, knee and ankle joint kinematics $(p<0.05)$. Peak knee flexion increased as sled loads increased $(p<0.05)$, which may enable athletes to lower their centre of mass and increase their horizontal force application. Net horizontal and propulsive impulse measures were greater in all sled conditions $(p<0.05)$, which increased significantly as sled loadings were heavier. In conclusion, this study highlights the effects of differential loads to help coaches understand acute kinetics and kinematic changes in order to improve the planning of sprint training.

Keywords: acceleration, biomechanics, kinematics, kinetics, sled towing

Word count: 3944 


\section{INTRODUCTION}

Sprint acceleration is defined as the capacity to generate as high a velocity as possible in as short a distance or time as possible (22), and is essential for success in the majority of sports $(14,29)$. In field sports, where the need to reach the ball first or be in position for play to develop is decisive, acceleration is a crucial factor $(22,29)$. Maximum velocity may not be as important as sprint acceleration in field sport players (29). The different sprint phases are regularly tested and monitored as they are considered key determinants of overall sprint performance (31). Research shows that rapid acceleration requires a powerful drive of the arms, hips and legs resulting in short contact times and an increased stride frequency $(24,29)$. Alternatively, other studies have placed a greater emphasis on a forward body lean (45 degrees), thereby increasing horizontal force application $(16,20)$. exercises (10), plyometric exercises (13) or with a more combined approach (9). Programmes are generally focussed on either increasing an athlete's maximal strength or power; however, coaches can also focus on movement efficiency or force application (7). These modalities may have a better transfer to performance compared to non-specific strength training (36). Resisted sprint training methods such as sled towing (ST), parachutes, weighted vests, bungees and uphill running offer the coach performed in a horizontal direction, and involve the relevant muscles, velocities and ranges of motion to those of uninhibited sprinting $(1,35)$. Research suggests that such sprint-specific training methods can lead to greater speed development (4). ST 
provides an external load in the form of a sled towed via a shoulder or waist harness and cord, behind the athlete. The mass of the sled and the friction coefficient between the sled and the ground surface affect external load and the subsequent impact on performance (21). Sleds are generally loaded based on a percentage of body mass $(\mathrm{BM})$ or percentage of velocity decrement $\left(\mathrm{V}_{\mathrm{Dec}}\right)(3,17,35)$. However, loadings based on a percentage BM do not account for individual variations in strength, power or technical ability. As such, loading sleds based on $V_{\text {Dec }}$ over a given distance is the preferred approach (31).

Acute ST studies are important as they allow researchers to investigate how different loading strategies can alter kinetics and kinematics. These acute changes may determine long-term adaptations. Sled loading strategies have varied greatly between studies, some researchers have investigated loads as light as 5\% BM (30) and others as heavy as $80 \%$ BM (27). Unsurprisingly, findings suggest that as sled loadings increased, sprint kinematics (velocity, contact time, stride length and stride frequency etc.) were changed to a greater extent $(23,25,30)$. As such, some investigations have recommended sled loadings of approximately $10 \% \mathrm{BM}$ or $10 \% \mathrm{~V}_{\mathrm{Dec}}$ in order to minimise the alterations to sprint kinematics (24). However, recent investigations have reported that moderate to heavy sled loadings may be required in order to provide an optimal overload for sprint acceleration (25). These loadings may increase horizontal ground reaction forces (GRF), which have been shown to be a key determinant of sprint acceleration (26). Kinetics and lower body kinematics have been explored over a range of different ST loads, despite numerous investigations $(18,24,30)$ there is little agreement on the optimum sled loading to develop the acceleration phase. 
76 The purpose of this study was to investigate kinetics and kinematics of ST during the early acceleration phase of sprinting in an elite academy rugby league population. Participants completed trials with a range of different sled loads $\left(10,15\right.$ and $\left.20 \% V_{\text {Dec }}\right)$ as well as uninhibited trials. It was hypothesised that (a) the disruption to lower limb and trunk kinematics would increase as sled loadings increased, (b) propulsive peak force would be greatest during the $20 \% V_{\text {Dec }}$ sled trials, and (c) propulsive impulses would be larger during the $20 \% V_{\text {Dec }}$ sled trials. The findings will allow coaches to understand the impact of different loading strategies and more accurately prescribe ST for the early acceleration phase.

\section{METHODS}

\section{Experimental Approach to the Problem}

This study used a randomised cross-over design to compare the effects of different ST loadings and uninhibited sprinting. Twelve rugby league athletes performed a series of $6 \mathrm{~m}$ sprints in four different conditions (Uninhibited, 10, 15 and 20\% $V_{\text {Dec }}$ ). The key dependant variables were the sagittal plane kinematic measures of the lower extremities and trunk, the kinetic data obtained from the force platform and various contact time measures.

\section{Subjects}

Twelve rugby league athletes from an elite academy (age: $18.9 \pm .6$ years; total body mass: $90.2 \pm 10.0 \mathrm{~kg}$; stature: $1.80 \pm 0.06 \mathrm{~m}$ ) participated in this study. All subjects were resistance trained ( $\geq 3$ years) with $S T$ experience and provided informed consent before attending the testing sessions. The Institutional Ethics Committee in 
accordance with the principles of the Declaration of Helsinki approved the testing procedures implemented in this study. No external funding was provided for this study.

\section{Procedures}

One week prior to testing, all subjects completed a familiarization session. The same sled was used throughout testing. The sled was attached to the subjects using a $3 \mathrm{~m}$ non-elasticated attachment cord and waist belt (See Figure 1). Using a $6 \mathrm{~m}$ uninhibited sprint as a baseline, sleds loadings (10,15 and 20\%) were determined in a random order. Sprint times were recorded using infrared timing lights (Smartspeed Ltd., Fusionsports, Queensland, Australia) and sled loadings were adjusted to reduce $6 \mathrm{~m}$ average velocity by the appropriate percentages (3). Mean sled loadings (sled plus additional load) based on \% $V_{\text {Dec }}$ and the equivalent $\% B M$ values are shown in table 1.

Measures were taken to ensure that no force plate targeting occurred. Firstly, the familiarization session was used to determine an individual starting position for each subject. Starting positions were adjusted so that each participant's right foot (dominant) contacted the force plate on their third step. Starting positions of the ST trials were also adjusted accordingly and practiced until participants could consistently 
land on the force plate. In order to standardise starting positions, trials began in a 3

127 point position. All participants chose to start with their left foot leading in the 3 point starting position. Regardless of the starting point, subjects sprinted a total distance of $6 \mathrm{~m}$.

Subjects were asked not to participate in any physical activity 24 hours before the testing session. The testing session began with a standardised warm-up consisting of jogging (5 min), dynamic stretching (5 min) and a number of short sprints building up to maximum intensity ( $4 \times$ submaximal and $2 \times$ maximal).

Previous research has shown that ST trials can impact on the kinematics of any subsequent uninhibited sprint trials (18). As such, the uninhibited sprint trials were completed before any of the sled trials $\left(10 \%, 15 \%\right.$ and $\left.20 \% V_{\text {Dec }}\right)$. Once the uninhibited sprint trials were complete, all subsequent ST trials were randomized. Testing procedures were identical to those described previously in the familiarisation section. All subjects had 3 min recovery between each of the sprint trials. Five trials were collected for each condition. Again, subjects sprinted a distance of $6 \mathrm{~m}$ in a $22 \mathrm{~m}$ lab. The surface friction coefficient $(\mu)$ of the lab $(\mu=0.41)$ was determined using methods developed by Linthorne \& Cooper (21). An embedded force platform, sampling at 1000 $\mathrm{Hz}$, was positioned at approximately $3 \mathrm{~m}$ from the start (model 9281CA; dimensions = $0.6 \times 0.4 \mathrm{~m}$, Kistler Instruments Ltd). In order for the trials to be deemed successful, the whole foot had to contact the force platform. Trials were discarded in cases where any part of the foot did not land the force platform. Sprint times were generated for every trial, and any trials in which sprint velocity deviated more than $\pm 5 \%$ of the initial 
trial in that condition were not used in the final analysis. In this instance, an extended recovery period of 4 min was implemented and trials were repeated.

An eight camera motion analysis system (Qualisys Medical AB, Goteburg, Sweden) was used to capture kinematic data at $250 \mathrm{~Hz}$. In order to determine stance leg kinematics of the trunk, thigh, shank, and foot segments, retro-reflective markers were placed on the following bony landmarks; the right calcaneus, $1^{\text {st }}$ metatarsal head, $5^{\text {th }}$ metatarsal head, medial malleolus, lateral malleolus, medial epicondyle, lateral epicondyle, acromion process (both), T12 and C7 (6). The trunk was tracked using markers at both acromion processes, as well as the T12 marker. The pelvis segment was defined, using additional markers on the anterior (ASIS) and posterior (PSIS) superior iliac spines. Hip joint centre was determined based on the Bell et al. (2) equations via the positions of the PSIS and ASIS markers. The ASIS, PSIS and greater trochanters were used as tracking markers for the pelvis. Rigid cluster tracking markers were also positioned on the right thigh and shank segments (5) Knee joint centre was delineated as the mid-point between the femoral epicondyle markers. The ankle joint centre was identified as the mid-point between the malleoli markers. During dynamic trials the foot segment was tracked using the calcaneus, $1^{\text {st }}$ and $5^{\text {th }}$ metatarsal heads. A static calibration was completed and used as reference for anatomical marker placement in relation to the tracking markers, after which all non-tracking markers were removed.

\section{Data Processing}

Motion files collected through the Qualisys track manager software and exported as C3D files and quantified using Visual 3-D (C-Motion Inc., Germantown, USA) and 
filtered with a cut-off frequency of $12 \mathrm{~Hz}$ using a Butterworth $4^{\text {th }}$ order filter to adequately suppress motion artefacts without inducing excessive smoothing of the traces $(12,34)$. Three dimensional kinematics of the lower extremities and trunk were calculated using an XYZ cardan sequence of rotations ( $\mathrm{X}$ represents the sagittal plane, $\mathrm{Y}$ represents the coronal plane and $\mathrm{Z}$ the transverse plane). The relevant segments (thorax, thigh, shank and virtual foot) and reference segments (pelvis, thigh and shank) were used to calculate joint angles of the trunk, hip, knee and ankle joints respectively. The stance phase was determined as time over which $20 \mathrm{~N}$ or greater of vertical force was applied to the force platform (32). Kinematic waveforms were time-normalised to $100 \%$ of the stance phase and then all processed trials were averaged. Various kinematic measures from the trunk, hip, knee and ankle joints were investigated: angle at foot-strike, angle at toe-off, peak angle, range of movement (ROM) from foot-strike to toe-off, and the relative ROM (the angular displacement from foot-strike to peak angle) (Rel ROM). Resultant velocity at toe-off was calculated using the vertical and horizontal centre of mass. These variables were extracted from each of the five trials for each joint, data were then averaged within subjects for a comparative statistical analysis.

Force plate data was collected through the Qualisys track manager software and exported to Visual 3-D (C-Motion Inc., Germantown, USA) for processing. The durations of the braking and propulsive phases were based on anterior and posterior horizontal GRF. Peak GRF was determined for the following components: vertical, braking, propulsive. Vertical impulse was calculated as the area under the vertical ground reaction force-time curve (using a trapezoidal function) minus body weight impulse over the time of ground contact. The braking and propulsive impulses were 
determined by integrating all the negative and positive values of horizontal GRF, respectively, over the time of ground contact $(18,19)$. Net horizontal impulse was calculated as propulsive impulse minus the absolute value of braking impulse. All impulse measures were normalised to body mass so they represent changes in velocity of centre of mass during ground contact (28). Similarly, mean values of vertical and net horizontal GRF were obtained by dividing respective impulse values by the contact time. Mean braking and propulsive GRF were calculated by dividing the respective impulse values by the time duration of the braking and propulsive phases, respectively (18). GRF measures were also normalised relative to body mass $(3,18)$.

\section{Statistical Analysis}

Descriptive statistics were calculated and presented as mean \pm standard deviation (SD). Dependant variables were examined using the uninhibited sprint trials. Testretest reliability and within-subject variation was evaluated using intraclass correlation coefficient (ICCs) and coefficients of variance (CV\%). Magnitudes of ICCs were classified according to the following thresholds: 0.9 nearly perfect; $0.7-0.9$ very large; 0.5-0.7 large; 0.3-0.5 moderate; and 0.1-0.3 small (15). One-way repeated measures ANOVAs were used to compare the means of the different conditions (Uninhibited, 10, 15 and $20 \% V_{\text {Dec }}$ ) with the different outcome measures (velocity, contact time, kinetics and kinematics). Post hoc pairwise comparisons were conducted on all significant main effects using a Bonferroni adjustment to control for type I error. Mauchly's test was used to confirm sphericity for each analysis. If the assumption of sphericity was violated, a Greenhouse-Geisser adjustment was used. Effect sizes were calculated using partial eta ${ }^{2}\left(p \eta^{2}\right)$, in accordance with Cohen (8) $p \eta^{2}=0.2$ considered small, $p \eta^{2}$ 
$224=0.5$ medium and $p \eta^{2}=0.8$ large. Significance levels were set at $p \leq 0.05$. All statistical analyses were undertaken using SPSS (Version 22, IBM SPSS Inc., Chicago, USA).

\section{RESULTS}

\section{Reliability of Measurement Variables}

230 Trials were monitored using sprint velocity which was shown to be reliable and have little variation across the population (ICCs $\geq 0.9 ; \mathrm{CV} \%=1.6)$. Range of ICCs and CV\% between participants and trials varied greatly among the other measurement variables (ranges shown after each section).

Figure 2 presents the mean sagittal plane angular kinematics during the stance phase.

\section{Velocity and Contact Time Measures}

Table 2 presents the stance phase contact time and velocity data. Velocity was reduced significantly in all sled conditions as loading increased $(p=0.001)$. Contact times increased significantly in all sled conditions as loading increased $(p<0.001)$. All sled conditions resulted in significantly greater propulsive times than uninhibited sprinting $(p<0.001)$, propulsive times increased with loading $(p<0.05)$. ICCs ranging between .47 (brake time) and .90 (velocity) were calculated. CV\% ranging between 1.6 (velocity) and $28.8 \%$ (brake time) were calculated. 


\section{Kinetic Measures}

255 The kinetic variables can be observed in Table 3. Vertical mean force during the $20 \%$ 256 loading condition was significantly lower than the uninhibited trials $(p=0.024)$. Net 257 horizontal mean force was greater in all ST conditions compared to the uninhibited 258 trials $(p<0.01)$. There was no significant difference between ST conditions $(p>0.05)$. 259 The propulsive mean force recorded during the $20 \%$ loading was significantly higher 260 than that of the uninhibited condition $(p=0.032)$. Again, there was no significant 261 difference between ST conditions $(p>0.05)$. Net horizontal and propulsive impulse 262 measures were significantly greater as sled loading increased $(p<0.05)$. ICCs ranging 263 between .22 (net horizontal impulse) and .66 (braking peak force) were calculated. 264 CV\% ranging between 6.9 (propulsive peak force) and 67.6\% (braking mean force) 265 were calculated.

266

\section{Trunk Kinematics}


273 The results (see Table 4) indicate that trunk angle at toe-off was significantly greater 274 during ST than the uninhibited trials $(p<0.05)$. There was no significant difference between ST conditions $(p>0.05)$. Relative trunk ROM was significantly greater in the

276

277

280

281

282

283

284

285

286

287

288

289

290

291

292

293

294

295

296

297 $20 \%$ loading condition compared to the uninhibited trials $(p=0.035)$. ICCs ranging between .68 (Rel ROM) and .94 (angle at foot-strike) were calculated. CV\% ranging between 7.4 (Rel ROM) and 16.1\% (ROM) were calculated.

@@@Table 4 inserted near here@@@

\section{Hip Joint Kinematics}

Hip joint measures can be observed in Table 5. ST had no significant impact on kinematics of the hip joint. ICCs ranging between .88 (peak flexion) and .94 (angle at toe-off) were calculated. CV\% ranging between 4.9 (peak flexion) and 30.7\% (angle at toe-off) were calculated.

(1)

(1)

$$
\text { @@@ Table } 5 \text { inserted near here @@@ }
$$

2

\section{Knee Joint Kinematics}

95 Knee joint measures can be observed in Table 5. Knee flexion at foot-strike was significantly greater as sled loading increased $(p<0.05)$. Similarly, peak flexion was greater as loading increased $(p<0.05)$. ROM in all ST conditions were significantly 
298 greater than the uninhibited trials $(p<0.01)$. ROM in the $20 \%$ sled loading condition 299 was also significantly greater than the $10 \%$ condition $(p=0.001)$. ICCs ranging between .63 (Rel ROM) and .82 (angle at toe-off) were calculated. CV\% ranging between 5.1 (peak flexion) and 20.1\% (ROM) were calculated.

\section{Ankle Kinematics}

The results (see Table 7) indicate that ankle ROM during ST conditions were significantly greater than the uninhibited trials $(p<0.05)$. There was no significant difference between ST conditions ( $p>0.05)$. ICCs ranging between .70 (angle at footstrike) and .94 (angle at toe-off) were calculated. CV\% ranging between 7.4 (angle at toe-off) and $21.0 \%$ (angle at foot-strike) were calculated.

\section{DISCUSSION}

To our knowledge, this is the first ST study to examine trunk and lower body 321 kinematics, contact time variables and kinetics during early acceleration in high-level 322 field sport athletes. Therefore, this study will provide a valuable insight for strength 
and conditioning coaches looking to prescribe ST (\% $\mathrm{V}_{\mathrm{Dec}}$ ) for field sport athletes. The major findings of this study were (a) as sled loadings increased trunk and lower extremity kinematics were altered to a greater extent, (b) there were no significant differences in propulsive peak force between any of the sled conditions and uninhibited sprinting, and (c) propulsive impulse measures in the $20 \% V_{\text {Dec }}$ sled trials were significantly greater than all other conditions.

In general, sprint kinematics were affected in all sled conditions when compared with uninhibited sprinting. This supports previous research $(3,18)$ and casts further doubt on the belief that lighter sled loadings $\left(10 \% \mathrm{BM}\right.$ or $10 \% \mathrm{VDec}_{\mathrm{De}}$ will not affect sprint kinematics. Previous investigations have suggested that when heavier sleds are utilised kinematic alterations to stride length and frequency are greater $(22,24,30)$. Although stride length and frequency were not measured in the present study, our results indicate that velocity and contact time were affected to a greater extent when sled loadings were increased. The longer contact times were explained by an extended propulsive phase, as suggested previously $(18,25,30)$. The additional contact time allows the athlete to exert greater propulsive forces to overcome the extra resistance provided by the sled. This increased propulsive contact time may be beneficial for acceleration performance, in this instance more horizontal force can be applied to the ground $(19,27)$.

ST with light to moderate loadings using a waist harness attachment appears to have no significant impact on hip joint kinematics. This finding differs from previous research by Monte et al. (25) who reported significant kinematic alterations at the hip, knee and ankle joints at foot-contact and take-off. However, the greater sled loadings utilised in 
348 their study (30 and 40\% BM) likely explains the difference. The only kinematic alterations observed at the ankle joint in the present study was a significantly lower ROM in the uninhibited condition compared to all ST trials. The change in ROM during sled trials was explained by a trend of increased dorsiflexion at foot-strike and increased plantarflexion at toe-off. Kinematic adjustments of this nature appear to allow the athletes to increase their stance phase contact times, as discussed previously. Our results show that there were a number of significant kinematic changes at the knee joint. Knee flexion at foot-strike and peak flexion were greater in all sled conditions and increased in line with loading. We believe these adjustments allow the athletes to lower their centre of mass and increase contact time, thus helping them overcome the added resistance of the sled by increasing their horizontal force application. Studies have highlighted the importance of trunk kinematics during ST and uninhibited sprinting alike $(3,19)$. Our results support this finding; extension of the trunk was significantly greater in the uninhibited condition compared to all sled conditions at toe-off. There was a trend for greater trunk flexion as sled loadings increased; however, this was not significant. Along with increased peak knee flexion, the authors believe the increased trunk flexion at toe-off enables the athlete to increase their horizontal force application. Adaptations of this nature have been reported after sled towing interventions, during acceleration such practice effects may lead to greater propulsive forces in the later stance phase $(1,19,35)$.

The authors hypothesised that propulsive peak force would be greatest in the $20 \%$ VDec sled condition. Results did not support this; there was however, a trend that as sled loading increased so too did propulsive peak force. It does appear that propulsive peak force would continue to increase with heavier sled loadings, as suggested in 
373 previous studies (27). It is important to note that such increases are at the expense of much greater contact times, which after a certain point may become counterproductive (24). Additionally, previous research suggests that the magnitude of forces may not be as important as the direction of force application $(19,26)$. Propulsive mean force was significantly higher and vertical mean force significantly lower in the $20 \% V_{\text {Dec }}$ sled condition. These kinetic changes again highlight the increased horizontal force vector orientation when towing moderate sled loads.

Net horizontal and propulsive impulses are key determinants of early acceleration $(16,19)$. However, simply maximising these measures at the expense of other key variables such as contact times may not be beneficial (19). Our results indicate that both net horizontal and propulsive impulses were significantly greater in all sled conditions and increased in line with sled loading. This supports the findings of previous investigations that utilised similar sled loading strategies (18). Again, the larger impulse measures reported can be explained by the increased contact times. As such, when rapid acceleration and shorter contact times are a priority $20 \% V_{\text {Dec }}$ sled towing may not be the ideal loading strategy, during these specific precompetition training periods uninhibited sprinting might be more appropriate. However, during the general preparation phase of training coaches may look to overload horizontal force application with this loading strategy. In this instance, ST may enhance the transition between high-strength and high-velocity exercises (1).

Unsurprisingly, heavier sled loadings led to a greater sprint velocity reduction (31). In the present study sled loadings were determined using $\% \mathrm{~V}_{\text {Dec }}$ rather than $\% \mathrm{BM}$. Sled loadings adjusted based on \% BM will not provide an optimal overload among all 
athletes because this method does not account for the athlete's muscular strength and sprint technique (18). Greater individual differences were apparent when towing heavier sleds, highlighted in this investigation by larger standard deviations as sled loadings increased. As such, it is recommended that coaches load sleds based on a $\% V_{\text {Dec }}$ rather than a \% $\mathrm{BM}$.

Investigations have demonstrated that females exhibit distinct lower body kinematics when compared with males (33). As such, the results are limited to this population and may not be applicable to female athletes. Similarly, the results are specific to the highly trained population and may not be applicable to recreational athletes. The light to moderate sled loadings utilised in this study may be a limitation. Researchers have recently suggested that very heavy sled loadings may provide the optimal training stimulus by maximising peak power output (11). It is beyond the scope of the present study to comment on such loading strategies.

\section{Practical Applications}

Overall, the results of this study have shown that a sled loading of $20 \% V_{\text {Dec }}$ enables coaches to increase propulsive forces and impulses. However, a blanket application of such loads may not be the most appropriate strategy as some of the acute changes are potentially counterproductive, such as reduced velocity and greatly increased contact times. Thus, perhaps a periodized approach should be adopted. For example, training with a $20 \%$ Vec sled loading will allow a greater emphasis on the horizontal application of forces then progressing to lighter sled loads or uninhibited sprint training to allow greater transfer of potential adaptations (e.g., maintain force/ impulse production whilst lowering contact times). The study therefore, highlights the effects of 
423 differential loads to help coaches understand acute biomechanical changes in order 424 to improve planning of sprint training.

\section{REFERENCES}

427

428 1. Alcaraz, PE, Elvira, JLL, and Palao, JM. Kinematic, strength, and stiffness 429 adaptations after a short- term sled towing training in athletes. Scand J Med Sci Sports, 24 (2): 279-290, 2014.

2. Bell, AL, Brand, RA, and Pedersen, DR. Prediction of hip joint centre location 432 from external landmarks. Hum Mov Sci, 8 (1): 3-16, 1989.

433 3. Bentley, I, Atkins, SJ, Edmundson, CJ, Metcalfe, J, and Sinclair, JK. Impact of 434 harness attachment point on kinetics and kinematics during sled towing. $J$ Strength 435 Cond Res, 30 (3): 768-776, 2015.

436 4. Brughelli, M, Cronin, J, and Chaouachi, A. Effects of running velocity on running 437 kinetics and kinematics. J Strength Cond Res, 25 (4): 933-939, 2010.

438 5. Cappozzo, A, Cappello, A, Croce, UD, and Pensalfini, F. Surface-marker 439 cluster design criteria for 3-D bone movement reconstruction. IEEE Transactions on $440 \quad$ Biomed Eng, 44 (12): 1165-1174, 1997.

441 6. Cappozzo, A, Catani, F, Leardini, A, and Benedeti, MG. Position and orientation 442 in space of bones during movement: Anatomical frame definition and determination. 443 Clin Biomech, 10 (4): 171-178, 1995.

444 7. Cissik, JM. Means and methods of speed training, part I. Strength Con J, 26 445 (4): 24-29, 2004.

446 8. Cohen J. Statistical power analysis for the behavioral sciences. New York, NY: 447 Routledge Academic, 1977. 
9. Comfort, P, Haigh, A, and Matthews, MJ. Are changes in maximal squat strength during preseason training reflected in changes in sprint performance in Rugby League players? J Strength Cond Res, 26 (3): 772-776, 2012.

451

10. Cormie, P, McGuigan, MR, and Newton, RU. Adaptations in athletic performance after ballistic versus strength training. J Sci Med Sport, 42 (8): 15821598, 2010.

454

11. Cross, MR, Brughelli, M, Samozino, P, Brown, SR, and Morin, JB. Optimal loadings for maximising power during sled-resisted sprinting. Int $J$ of Sports Physiol Perf, 12 (8): 1-25, 2017.

12. Debaere, S, Delecluse, C, Aerenhouts, D, Hagman, F, and Jonkers, I. From block clearance to sprint running: Characteristics underlying an effective transition. $J$ Sports Sci, 31 (2): 137-149, 2013.

13. De Villarreal, ES, Requena, B, and Cronin, JB. The effects of plyometric training on sprint performance: A meta-analysis. J Strength Cond Res, 26 (2): 575-584, 2012. 14. Duthie, GM, Pyne, DB, Marsh, DJ, and Hooper, SL. Sprint patterns in rugby union players during competition. J Strength Cond Res, 20 (1): 208-214, 2006.

15. Hopkins, WA. Scale of magnitudes for effect statistics [Internet]. In: A New View of Statistics Internet Society for Sport Science. Available at: http://www.sportsci.org/resource/stats/index.html, 2002. Accessed January 14, 2018. 16. Hunter, JP, Marshall, RN, and McNair, PJ. Relationships between ground reaction force impulse and kinematics of sprint-running acceleration. J Appl Biomech, $21(1): 31-43,2005$.

17. Kawamori, N, Newton, RU, Hori, N, and Nosaka, K. Effects of Weighted Sled

471 Towing With Heavy Versus Light Load on Sprint Acceleration Ability. J Strength Cond 472 Res, 28 (10): 2738-2745, 2014. 
473 18. Kawamori, N, Newton, RU, and Nosaka, K. Effects of weighted sled towing on 474 ground reaction force during the acceleration phase of sprint running. J Sports Sci, 32 $475 \quad(12): 1-7,2014$.

19. Kawamori, N, Nosaka, K, and Newton, R. Relationships between ground 477 reaction impulse and sprint acceleration performance in team sport athletes. $J$ Strength Cond Res, 27 (3): 568-573, 2013.

20. Kugler, F. and Janshen, L. Body position determines propulsive forces in accelerated running. J Biomech, 43 (2): 343-348, 2010.

21. Linthorne, NP, and Cooper, JE. Effect of the coefficient of friction of a running surface on sprint time in a sled-towing exercise. Sports Biomech, 12 (2): 175-185, 2013.

22. Lockie, RG, Murphy, AJ, Schultz, AB, Jeffriess, MD, and Callaghan, SJ. Influence of sprint acceleration stance kinetics on velocity and step kinematics in field sport athletes. J Strength Cond Res, 27 (9): 2494-2503, 2013.

23. Lockie, RG, Murphy AJ, and Spinks, CD. Effects of Resisted sled towing on sprint kinemtaics in field-sport athletes. J Strength Cond Res, 17 (4): 760-767, 2003. 24. Maulder, PS, Bradshaw, EJ, and Keogh, JW. Kinematic alterations due to different loading schemes in early acceleration sprint performance from starting blocks. J Strength Cond Res, 22 (6): 1992-2002, 2008.

25. Monte, A, Nardello, F, and Zamparo, P. Sled towing: The optimal overload for peak power production. Int J Sports Physiol Perf, 12 (8): 1052-1058, 2017. 26. Morin, JB, Edouard, P, and Samozino, P. Technical ability of force application as a determinant factor of sprint performance. Med Sci Sports Exerc, 43 (9): 16801688, 2011. 
497 27. Morin, JB, Petrakos, G, Jiménez-Reyes, P, Brown, SR, Samozino, P, and 498 Cross, MR. Very-heavy sled training for improving horizontal force output in soccer players. Int J Sports Physiol Perf, 12 (6): 840-844, 2017.

28. Mullineaux, DR, Milner, CE, Davis, IS, and Hamill, J. Normalization of ground reaction forces. J App Biomech, 22: 230-233.

29. Murphy, AJ, Lockie, RG, and Coutts, AJ. Kinematic determinants of early acceleration in field sports athletes. J Sports Sci Med, 2 (4): 144-150, 2003.

30. Murray, A, Aitchison, TC, Ross, G, Sutherland, K, Watt, I, McLean, D, and

Grant, S. The effect of towing a range of relative resistances on sprint performance. $J$ Sports Sci, 23 (9): 927-935, 2005.

31. Petrakos, G. Morin, JB, and Egan, B. Resisted sled sprint training to improve sprint performance: A systematic review. Sports Med, 46 (3): 381-400, 2016.

32. Sinclair, JK, Edmundson, CJ. Brooks, D. and Hobbs, SJ. Evaluation of kinematic methods of identifying gait events during running. Int J Sports Sci Eng, 5 (3): 188-192, 2011.

33. Sinclair, JK, Greenhalgh, A, Edmundson, CJ, and Brooks, D. Gender differences in the kinetics and kinematics of distance running: Implications for footwear design. Int J Sports Sci Eng, 6 (2): 118-128, 2012.

34. Slawinski, J, Dumas, R, Cheze, L, Ontanon, G, Miller, C, and MazureBonnefoy, A. Effect of postural changes on 3D joint angular velocity during starting block phase. J Sports Sci, 31 (3): 256-263, 2013.

35. Spinks, CD, Murphy, AJ, Spinks, WL, and Lockie, RG. The effects of resisted sprint training on acceleration performance and kinematics in soccer, rugby union, and Australian football players. J Strength Cond Res, 21 (1): 77-85, 2007. 
521 36. Young, WB. Transfer of strength and power training to sports performance. Int $522 J$ Sports Physiol Perf, 1 (2): 74-83, 2006.

523

524

525

526

527

528

529

530

531

532

533

534

535

536

537

538

539

540

541

542

543

544

545 
546 Figure labels

547 Figure 1. The sled, cord and harness attachment.

548 Figure 2. Mean trunk (a) hip (b) knee (c) and ankle (d) joint angles in the sagittal

549 plane for the uninhibited (bold black line), 10\% (bold grey line), 15\% (dashed black 550 line) and $20 \%$ (dotted grey line) conditions. 